\title{
Du Syndicalisme dans les Structures Professionnelles des Eglises à Bukavu (République Démocratique du Congo)
}

\author{
Habamungu Bashwira, \\ Chef de Travaux à l'UOB/ Département de Sociologie, \\ République Démocratique du Congo \\ Pilo Kamaragi, \\ Professeur à l'Institut Supérieur Pédagogique de Bunia, \\ République Démocratique du Congo
}

Doi:10.19044/esj.2020.v16n22p118 URL:http://dx.doi.org/10.19044/esj.2020.v16n22p118

\section{Résumé}

La présente étude porte sur le syndicalisme dans les structures professionnelles des Eglises de la Ville de Bukavu. Il s'agit de circonscrire les fonctions, les perceptions et les contraintes des associations syndicales dans les services promus par les Eglises à Bukavu. Pour la collecte des données, 401 sujets issus de 42 structures professionnelles des Eglises de Bukavu, sélectionnées sur base de leur capacité élevée de production et du nombre (30 travailleurs et plus) du personnel pris en charge ont été enquêtés à l'aide du questionnaire et de l'entretien. La méthode structuro-fonctionnaliste de T. Parsons (1973), l'analyse statistique et le test chi carré ont soutenu le dépouillement et le traitement des données. Il ressort des résultats de la recherche que les syndicats sont en œuvre pour revendiquer les bonnes conditions de travail. L'on assiste à des mouvements des revendications allant des avertissements de grèves par des lettres adressées directement aux autorités religieuses et politico-administratives, «set in », à des cessations partielles de travail dans beaucoup d'entreprises. Toutefois les syndicats œuvrant dans les structures professionnelles des Eglises sont diversement appréciés par les employés et les employeurs. Pour les uns, les syndicats existent théoriquement mais ne remplissent pas leur rôle. Pendant que pour les autres, les syndicats travaillent dans l'intérêt général des agents. Dans la poursuite de leurs missions, les syndicats se heurtent à des obstacles de divers ordres. Tout d'abord, ils sont l'émanation des institutions religieuses qui digèrent difficilement les oppositions au sein de leurs structures. Ensuite, les syndiqués sont souvent dubitatifs dans les démarches revendicatives. Ils ont peur de s'engager dans les démarches craignant la perte de leurs emplois. Enfin, les syndicalistes sont eux-mêmes souvent inféodés au pouvoir car 
dépendant de la ligne droite définie par certains responsables des structures professionnelles des Eglises.

Mots clés : Luttes syndicales, structures professionnelles, Eglises, Emplois, Bukavu

\title{
Labor Unionism in Church Owned Professional organizations of Bukavu
}

\author{
Habamungu Bashwira, \\ Chef de Travaux à l'UOB/ Département de Sociologie, \\ République Démocratique du Congo \\ Pilo Kamaragi, \\ Professeur à l'Institut Supérieur Pédagogique de Bunia, \\ République Démocratique du Congo
}

\begin{abstract}
This study deals with labor unionism in Church Owned Professional Organizations of the City of Bukavu. The paper aims at (1) identifying the actions of the labor unions, (2) examining how those actions are socially perceived and (3) finding out the challenges accounted by the labor unions in the Church Owned Organizations in Bukavu. 401 employees from 42 Church owned Professional Organizations in Bukavu were sampled and surveyed. The structural-functional method, the content analysis and the chi-square test supported the data analysis. The results of the research show that the labor unions are active in bargaining for improved employees' working conditions. There are several actions ranging from warnings of strikes through letters addressed directly to religious and politico-administrative authorities, to partial layoffs in many organizations. However the actions of the labor union members are differently perceived and appreciated by the employees and the employers. For some, the unions exist theoretically but do not fulfill their role. While for the others, the unions serve the general interest of the employees. In the pursuit of their missions, the labor unions always face several challenges. First, they operate in religious institutions which find it difficult to comply with opposition based view within their organization. Second, employees are often afraid and reluctant to raise their voice about their working conditions since this can result in job dismissal; thus, the process of defending their rights is a bit challenging than it would be in a different organization. Finally, the
\end{abstract}


labor unionists who are supposed to defend employees' rights are themselves often obedient to employers' case. Thus, they often comply with the straight line defined by the leaders of the Church Owned Professional organizations.

Keywords: Employment, Union, claim, churches, Rights

\section{Introduction}

L'analyse sociologique du syndicalisme et du mouvement ouvrier en général était jusqu'aux années 60 peu avancée. Les enquêtes sur le terrain, les études empiriques, sont rares, sauf aux Etats-Unis. Quant aux réflexions théoriques, elles étaient plus souvent le fait des juristes et d'essayistes politiques que des sociologues.

De nos jours, certes, on s'intéresse à nouveau et de plus en plus au rôle et au fonctionnement des organisations syndicales. L'initiative, partie des Etats-Unis, commence à se répandre dans les autres pays occidentaux et en Afrique. Ce domaine, longtemps abandonné aux spécialistes des relations industrielles, aux économistes et aux historiens sociaux, attire maintenant les sociologues et les psychosociologues. (Michel Crozier, 1963).

Si le travail est une obligation, c'est-à-dire un devoir, il est aussi en même temps une source de droits pour le travailleur. Les droits humains qui découlent du travail rentrent précisément dans l'ensemble plus large des droits fondamentaux de la personne. Sur le fondement de tous ces droits et en relation avec la nécessité où sont les travailleurs de les défendre eux-mêmes, se présente un autre droit : le droit d'association, c'est-à-dire le droit de s'associer, de s'unir, pour défendre les intérêts vitaux des hommes employés dans les différentes professions. Ces unions portent le nom de syndicats. Les intérêts vitaux des travailleurs sont, jusqu'à un certain point, communs à tous ; cependant, chaque genre de travail, chaque profession a une spécificité propre, qui devrait se refléter de manière particulière dans ces organisations.

Droz J. (1997) signale qu'il y a une nette distinction entre les corporations et le syndicat. L'histoire du socialisme dans l'immédiat aprèsguerre a été dominée par deux ordres des faits apparemment contradictoires : d'une part la consolidation inattendue de la révolution soviétique, d'autre part l'échec des mouvements révolutionnaires en Europe même.

L'expérience historique apprend que les organisations syndicales sont un élément indispensable de la vie sociale, particulièrement dans les sociétés modernes industrialisées. Cela ne signifie évidemment pas que seuls les ouvriers de l'industrie puissent constituer des associations de ce genre. Les représentants de toutes les professions peuvent s'en servir pour défendre leurs droits respectifs. En fait, il y a des syndicats d'agriculteurs et des syndicats de travailleurs intellectuels ; il y a aussi des organisations patronales. Ils se 
subdivisent tous en groupes et sous-groupes selon les spécialisations professionnelles.

Déjà en 1929, dans une lettre adressée au cardinal Liénart, l'Eglise catholique estime moralement nécessaire la constitution des associations syndicales. On a pu croire, un temps, que l'union organique des chefs d'entreprise et des ouvriers pourrait se réaliser au sein de l'association de base, le syndicat qu'on appelait alors «mixte ». Mais avec le temps, la formule du syndicat séparé a prévalu.

Le premier syndicat congolais fut créé par le décret du 18 janvier 1920. C'était l'unique syndicat et il était réservé aux Blancs : 1'Association des Fonctionnaires et Agents de la Colonie (A.F.A.C.). Pour Ollandet (2014), ce syndicat exclut les étrangers, c'est-à-dire, les non Belges, et les salariés européens de secteur privé.

L’ordonnance-loi n¹23 du 16 avril 1942, légiféra sur les unions professionnelles, minutieusement réglementées et réservées, une fois de plus, exclusivement aux non africains. Le 29 juin 1944, un véritable mouvement syndical unitaire fut créé. En effet, toutes les associations réalisèrent une fusion totale pour donner naissance à la Confédération Générale des Syndicats du Congo (CGS).

Sous l'influence de la guerre de 1939-1945 et du syndicalisme travailliste anglo-saxon, la CGS revêtit rapidement un caractère politique révolutionnaire, flétrit le régime parlementaire, le capitalisme, et prôna la nationalisation des principaux secteurs économiques du pays.

Dans ce contexte, le syndicalisme africain s'est érigé en principal mouvement de lutte pour les indépendances et a constitué une pépinière de cadres formés en vue de l'émancipation de l'Afrique (Fonteneau et al. 2004).

La constitution de la République Démocratique Congo (2006), dans le Titre III relatif aux Droits fondamentaux et devoirs des citoyens reconnait à ces derniers, le droit de constituer des associations et des sociétés. Elle reconnait aussi aux travailleurs le droit de grève, quitte à l'exercer dans le cadre de l'action syndicale et conformément aux lois. C'est ainsi que le monde du travail congolais entra dans une phase : celle du pluralisme syndical.

La caractéristique du travail est avant tout d'unir les hommes et c'est en cela que consiste sa force sociale : la force de construire une communauté.

Léon XIII a proclamé solennellement le droit d'association que la révolution française avait refusé à l'ouvrier en ces termes : «les sociétés privées n'ont d'existence qu'au sein de la société civile dont elles sont autant de parties. Il ne s'ensuit pas cependant qu'il soit au pouvoir de l'Etat de leur dénier l'existence. Le droit à l'existence leur a été octroyé par la nature ellemême et la société civile a été instituée pour protéger le droit naturel, non pour l'anéantir. ") Ainsi, le Pape des ouvriers reconnaissait-il l'association professionnelle de son temps (syndicat ouvrier et le syndicat mixte), de droit 
naturel, c'est-à-dire conforme aux besoins de la nature humaine (Rollet $\mathrm{H}$. 1959).

C'est un procédé que la doctrine sociale catholique reconnaît comme légitime sous certaines conditions et dans des justes limites. Les travailleurs devraient se voir assurer le droit de grève et ne pas subir de sanctions pénales personnelles pour leur participation à la grève. Tout en admettant que celle-ci est un moyen juste et légitime, on doit également souligner qu'elle demeure, en un sens, un moyen extrême.

Cet article analyse la dynamique syndicale dans les structures professionnelles des Eglises de la Ville de Bukavu. Eu égard à cela, le présent article part des questionnements ci-après : Quelles sont les fonctions remplies par les syndicats au sein des structures professionnelles religieuses à Bukavu ? Comment ces fonctions sont-elles perçues par les ouvriers catholiques et protestants (de la $5^{\text {ème }}$ CELPA et $8^{\text {ème }}$ CEPAC) à Bukavu ? Quelles sont les contraintes auxquelles se heurtent les syndicats dans l'exercice de leurs fonctions au sein des structures professionnelles des Eglises à Bukavu ?

Pour répondre à ces questionnements, les hypothèses suivantes sont émises :

- La fonction essentielle des syndicats dans les services promus par les Eglises, serait la négociation collective, c'est-à-dire la discussion des salaires, des conditions de travail et éventuellement de tout problème pouvant s'y rattacher. Cependant, ce problème n'est pas clairement accepté dans toutes les entreprises confessionnelles de la même manière.

Le système des négociations collectives constitue également, à ces jours, le moyen d'action le plus élaboré dont dispose le mouvement ouvrier. Ces négociations évoluent différemment jusqu'à la mise en place, dans certaines entreprises confessionnelles, des conventions collectives entre employeurs et syndicats.

- Les ouvriers du secteur religieux auraient une double perception des actions syndicales dans leurs services. Pour les uns, les syndicats existeraient théoriquement mais ne remplissent pas leur rôle pendant que pour d'autres les syndicats travailleraient dans l'intérêt général des employés.

- La soumission étroite à la logique du chef, les mécanismes de recrutement appliqués, les motivations diversifiées des employés du secteur des Eglises et le patronat constitué essentiellement des membres des Eglises sont les contraintes majeures auxquelles se heurtent les syndicats du secteur professionnel religieux.

Cette recherche poursuit les objectifs de $: 1^{\circ}$ ) dégager les actions de la délégation syndicale $; 2^{\circ}$ ) circonscrire les perceptions que les acteurs sociaux se font des réalisations des délégations syndicales dans leurs entreprises et $3^{\circ}$ ) 
déceler les contraintes des associations syndicales dans les services promus par les Eglises à Bukavu.

Outre l'introduction, la conclusion et la bibliographie, la présente recherche comprend quatre sections. La première section porte sur la présentation du champ d'étude ; pour sa part, la deuxième section met en évidence les matériels et la méthodologie de la recherche ; la troisième section quant à elle s'attarde sur les considérations théoriques sur le syndicalisme et la quatrième section enfin, s'attèle sur la présentation et la discussion des résultats de la recherche.

\section{Champ d'etude}

Le souci de travailler en profondeur et de ne pas papillonner conduit à délimiter cette recherche dans le temps et dans l'espace.

Sur le plan temporel, la présente recherche porte sur la période allant de l'année 2001 à l'année 2019, soit 19 ans. Cette période est caractérisée non seulement par une crise multiforme du système social congolais allant des guerres, des crises économiques, d'une forte croissance démographique occasionnée par l'exode rural de plusieurs habitants en fuite de l'insécurité grandissante, mais aussi à la multiplication des confessions religieuses en quête de fidèles et adeptes.

Sur le plan spatial, les investigations se limitent aux confessions religieuses de la Ville de Bukavu, plus précisément l'Eglise Catholique, la 5 ème CELPA et la $8{ }^{\text {ème }}$ CEPAC. Ces Eglises ou communautés religieuses ont créé des emplois dans divers secteurs de la vie (éducatif, sanitaire, paroissial, philanthropique ou humanitaire, agro-pastoral).

L'on constate actuellement partout dans la Ville de Bukavu des œuvres sociales de l'Eglise Catholique, de la $5^{\text {ème }}$ CELPA et de la $8^{\text {ème }}$ CEPAC. Ces Eglises contrôlent une main-d'œuvre non négligeable dans les secteurs sanitaire et scolaire, dans les organisations caritatives, associatives et sociales.

La Ville de Bukavu est située à l'Est de la République Démocratique du Congo à 28 $8^{\circ} 1^{\prime}$ de longitude Est et à une altitude moyenne de $1600 \mathrm{~m}$. C'est la ville la plus élevée du pays. Chef-lieu de l'ancienne Province du Kivu, Bukavu a été créé par l'ordonnance N²2/1396 du 29/06/1958 qui en fixe les limites. La Ville de Bukavu est devenue la capitale de la Province du SudKivu, par l'ordonnance-loi N`88-031 du 20 juillet 1988 portant découpage territorial divisant l'ancienne Province du Kivu en trois provinces distinctes : celle du Maniema, celle du Nord-Kivu et celle du Sud-Kivu. (Pilo \& Rugambwa, 2005).

Cette ville est limitée au Nord par le Lac Kivu, au Sud et à l'Ouest par le Territoire de Kabare et à l'Est par la rivière Ruzizi qui forme une frontière naturelle avec le Rwanda. Elle compte 1.184.955 habitants nationaux et 
étrangers en 2017installés sur une superficie de 44,90 $\mathrm{Km}^{2}$. (Mairie de Bukavu, Rapport annuel 2017).

Sur le plan professionnel, Cishunguluka, K. A. (2017) note qu'il existe dans la Ville de Bukavu des travailleurs et des chômeurs, des actifs et des passifs sociaux. C'est une ville où les études et, de surcroît, les diplômes sont perçus comme la clé de voute pour l'accès à l'emploi. Les études y sont considérées à la fois comme une arme d'intégration socioprofessionnelle et un prestige social.

Sur le plan religieux, la ville de Bukavu est majoritairement chrétienne. Pour l'Institut National des Statistiques (2017) la religion chrétienne est la première religion à Bukavu avec $91 \%$. Cette ville renferme beaucoup de confessions religieuses à savoir catholique, protestante, anglicane, Kimbanguiste, Musulmane, témoins de Jéhovah, etc. qui créent et gèrent des emplois. Les données recueillies à la division provinciale de la justice dans la Ville de Bukavu montrent qu'entre 2007 et 2015, le bureau chargé des associations sans but lucratif et des établissements d'utilité publique a enregistré 643 Associations confessionnelles dont 69 seulement, soit 10,73\% sont dotées de la personnalité juridique.

De nombreuses Eglises, surtout celles dites de réveil, fonctionnent sans se doter de la personnalité juridique pendant que l'article 47, alinéa $2^{\text {ème }}$ de la loi $n^{\circ} 004 / 2001$ du 20 juillet 2001 dispose que « Nul ne peut percevoir des dons, présents, legs ou aumônes au nom d'une association confessionnelle n'ayant pas la personnalité juridique ou l'autorisation de fonctionnement ».

Il est vrai que ces Eglises s'appuient sur l'article 22 de la constitution de 2006 de la République Démocratique du Congo qui stipule que «Toute personne a droit à la liberté de pensée, de conscience et de religion. Toute personne a le droit de manifester sa religion ou ses convictions, seule ou en groupe, tant en public qu'en privé, par le culte, l'enseignement, les pratiques, l'accomplissement des rites et l'état de vie religieuse sous réserve du respect de la loi, de l'ordre public, des bonnes mœurs et des droits d'autrui... »

Il est difficile de dégager le nombre exact de membres des Eglises protestantes de réveil au Congo, en général, et dans la Ville de Bukavu, en particulier, pour des raisons multiples. Leurs membres sont mobiles et souvent adeptes de plusieurs Eglises à la fois. (Lecuyer, P. et al. 2003)

En effet, les recherches de terrain montrent que certains membres sont souvent à la recherche de l'argent, des hommes et/ou des femmes, mais rarement de Dieu. Pour ce genre de fidèles, l'Eglise offre un bon cadre pour solliciter l'emploi ou un avantage auquel on n'aurait pas d'accès sans adhérer à un mouvement religieux. 


\section{Materiels et Methode}

Le choix de la méthode structuro-fonctionnaliste de T. Parsons (1973), s'est avérée indispensable dans cette recherche en vue d'identifier les soussystèmes qui interagissent dans le contexte des emplois du secteur religieux afin de faciliter l'insertion professionnelle des chômeurs et la rémunération décente dans les services promus par les Eglises de Bukavu. Cet article a recouru à l'approche comparative pour établir les ressemblances et les différences entre les organisations syndicales des travailleurs, le degré de satisfaction des agents au sein des structures professionnelles créées et gérées par Eglises de la Ville de Bukavu.

Pour la collecte des données, la présente recherche a mobilisé le questionnaire (qui a été administré à 401 employés) et l'entretien semi-directif (42 guides d'entretien ont été administrés aux 42 chefs de services concernés en vue de compléter les données fournies par les employés). Le dépouillement et l'interprétation des données qualitatives ont été facilités par l'analyse de contenu alors que le logiciel SPSS $\mathrm{N}^{\circ} 020$, la statistique et le Khi2 ont servi dans l'interprétation des données quantitatives. Ce test (le khi carré) a été privilégié dans la mesure où il fallait repérer la relation de dépendance ou d'indépendance entre les différentes variables. Pour l'opérationnalisation de la recherche, le recours à l'échantillon probabiliste stratifiée s'est avéré indispensable. La taille de l'échantillon est de 401 sujets issus de 42 structures professionnelles des Eglises de Bukavu, sélectionnées sur base de la capacité productive de leurs activités et du nombre (plus de 30) du personnel pris en charge.

Avant d'aborder l'analyse et l'interprétation des résultats, cet article présente à ce stade les différentes dimensions de l'analyse du syndicalisme.

\section{Considerations Theoriques sur le Syndicalisme}

Crozier (1963) distingue cinq points de vue complémentaires qui correspondent aux aspects divers et parfois contradictoires du mouvement ouvrier. Il s'agit de :

1. Le point de vue génétique : à quels besoins répond le mouvement ouvrier ? Ce sont bien sûr les historiens qui y ont répondu. Deux types d'interprétation historique ont jusqu'à présent dominé le mouvement ouvrier.

La première de cette interprétation consiste dans le fait que le mouvement ouvrier est la création volontaire et rationnelle d'un petit nombre d'animateurs qui ont su rassembler autour d'eux les meilleurs de la masse ouvrière.

L'interprétation marxiste orthodoxe constitue la seconde tendance qui a dominé en France l'étude du mouvement ouvrier. Marx (1965) met l'accent sur l'environnement technologique dans l'explication de l'origine du mouvement ouvrier, mais cette explication reste réductive car à côté de 
l'environnement technologique disent Friedmann et Naville (1972), il y a d'autres types d'environnement dans lesquels se sont développés les mouvements ouvriers intéressants.

2. Le point de vue structurel : quelle sorte d'organisation le mouvement ouvrier est-il ? Comment s'y répartissent les rôles et les pouvoirs ? Ce point de vue met en évidence l'exigence démocratique, or celle-ci ne peut être réalisable que dans un contexte socio-culturel particulier, alors qu'on la considère généralement comme un absolu-quelle que soit d'ailleurs l'option idéologique qu'on a prise.

En fait, la tâche de la recherche dans ce domaine est tout d'abord de clarifier cette notion de la démocratie dans le cadre d'une organisation volontaire, mais à forte tendance contraignante comme le mouvement ouvrier.

3. Le point de vue fonctionnel : quelles sont les fonctions du mouvement ouvrier dans la société globale et comment les remplit-il ? Nous avons décrit très minutieusement les procédures des négociations collectives, étudié leur influence sur la vie économique, analysé la situation légale des syndicats et le droit de négociations collectives.

Allant plus loin, nous avons démontré comment l'intervention des syndicats dans la vie professionnelle était en train de créer un droit nouveau.

4. Le point de vue idéologique : pourquoi l'importance du levier idéologique dans le mouvement ouvrier est considérable ? L'idéologie a une importance considérable dans un mouvement qui met en cause les fondements de l'organisation sociale. Certes, les syndicats ne sont pas des mouvements d'idées, mais ils n'ont pu se développer qu'en utilisant des armes idéologiques.

5. Le point de vue du changement : le mouvement ouvrier n'est pas seulement une réaction à une situation donnée, une des pièces indispensables de l'équilibre social, c'est aussi un facteur original nouveau qui constitue pour la société dans son ensemble un facteur de changement.

Cet article n'a pas la prétention d'aborder toutes les dimensions énumérées ci-haut au regard des exigences reconnues à ce genre de travail, mais il se contente d'interroger la pratique sur les aspects structurels et fonctionnels du mouvement syndical comme le souligne déjà la méthode utilisée.

\section{RESULTATS}

Comme souligné dans les lignes précédentes, cet article poursuit trois objectifs et partant, trois hypothèses. Cette section présente et interprète les 
résultats des enquêtes selon les hypothèses formulées au début de la recherche. Il s'agit concrètement de circonscrire les fonctions, les perceptions et les contraintes du mouvement syndical dans les structures professionnelles des Eglises de la ville de Bukavu.

\section{IV.1. Les fonctions de la délégation syndicale dans la ville de Bukavu}

Avant d'entrer dans le vif du sujet, il est impérieux de dire un mot sur l'importance accordée ou non aux syndicats par les enquêtés, la structure d'une délégation syndicale et les mécanismes de revendications développées par les syndicats.

\subsection{Importance des syndicats dans une structure professionnelle des Eglises}

Contre les abus de l'individualisme foncier du libéralisme et contre une socialisation qui, pour tout prévoir et tout organiser, s'oppose à la personne humaine et violente les consciences, le syndicalisme apparaît comme une nouvelle organisation professionnelle qui ordonnance et encourage les ouvriers à s'associer et à poursuivre leurs intérêts par des voies légitimes.

Pour se rendre compte de l'effectivité du mouvement syndical dans les structures professionnelles à Bukavu, la question concernant l'existence ou pas des délégations syndicales au sein des services promus par les Eglises a été posée aux enquêtés.

Tableau 1. Relation confession religieuse et présence de la délégation syndicale

\begin{tabular}{|c|r|r|r|r|}
\hline \multirow{2}{*}{$\begin{array}{c}\text { DELEGATION } \\
\text { SYNDICALE } \\
\text { DANS LE } \\
\text { SERVICE }\end{array}$} & \multicolumn{2}{|c|}{ CONFESSION RELIGIEUSE } & \multicolumn{2}{c|}{ TOTAL } \\
\cline { 2 - 5 } & PROTESTANT & CATHOLIQUE & FREQUENCE & $\%$ \\
\hline NON & $86(43.2 \%)$ & $101(50 \%)$ & 187 & 46,6 \\
\hline OUI & $113(56.8 \%)$ & $101(50 \%)$ & 214 & 53,3 \\
\hline TOTAL & 199 & 202 & 401 & 100 \\
\hline
\end{tabular}

Source : Enquête de terrain, Juillet-septembre 2019

Les pourcentages contenus dans ce tableau sont calculés en ligne comme en colonne. En ligne, ils ont été calculés en tenant compte des spécificités de chaque confession religieuse (199 et 202). Et en colonne, particulièrement le total, ces pourcentages sont calculés en fonction du total de l'échantillon, soit 401 enquêtés.

Deux positions se dégagent des données contenues dans ce tableau. La première ne reconnait pas l'existence des syndicats dans leurs emplois. D'après les tenants de cette position, les syndicats sont, la plupart de fois, étouffés par les responsables des services confessionnels qui ne leur laissent pas la marge de manœuvre pour bien s'acquitter de leurs tâches syndicales. 
C'est ainsi que certaines délégations syndicales commencent avec pompe, mais par manque de liberté, finissent par se taire. Les gestionnaires jouent ainsi un rôle capital dans l'existence des représentations des syndicats dans les emplois à Bukavu. Deux tendances contradictoires des gestionnaires sont visibles. La première tendance soutient que la représentation syndicale dans un service est un atout important pour l'épanouissement du personnel alors que la seconde tendance s'insurge contre la présence de la délégation syndicale dans une structure professionnelle. Selon cette dernière tendance, le syndicat est un facteur perturbateur de la paix au sein d'un service.

La deuxième position qui se dégage du tableau reconnait la présence des syndicats au sein des structures professionnelles. Ces enquêtés reconnaissent l'existence de deux types de syndicats dans la ville de Bukavu. Il s'agit des syndicats ouvriers et des syndicats patronaux. Les syndicats ouvriers : ces syndicats, dont la constitution leur paraît moralement nécessaire, l'Eglise les souhaite chrétien. Ce souhait, il faut l'entendre, à la limite, comme celui d'un syndicat suscité par des catholiques pour des catholiques et composé des catholiques. Même dans ce cas limite, il ne s'agit pas à proprement parler d'une association confessionnelle puisque le syndicat peut toujours recevoir des adhérents non chrétiens et qu'il prend librement ses options dans son domaine temporel. Dans l'Encyclique « singulari quadam ». Pie $\mathrm{X}$ note qu'il est nécessaire d'établir et de favoriser de toute manière ce genre d'association dans les pays catholiques et partout où il paraitra possible par elles de subvenir aux besoins des divers associés (Rollet, H.,1959).

Les premiers syndicats ouvriers témoignaient en France d'une si grande hostilité à l'Eglise. Le système monopolistique de l'Union Nationale des Travailleurs du Zaïre «UNTZA » a eu comme corollaires, dans le vécu quotidien du travailleur : l'inflation galopante, la dollarisation des biens et services, la non indexation des salaires au coût de la vie, le retard du paiement des salaires dans les entreprises, la perte considérable du pouvoir d'achat, la répression des mouvements de grève.

Le retour au pluralisme syndical constitue de ce fait une opportunité pour les travailleurs congolais.

Actuellement, dans la ville de Bukavu, trois types de syndicats posent problème à la conscience chrétienne, il s'agit des syndicats interconfessionnel, neutre et unique. Parmi ces syndicats, on cite le syndicat des écoles catholiques (SYNECAT), le Syndicat Libre Chrétien du Congo (SLCC) et le syndicat des écoles protestantes (SYNEP) qui fonctionnent différemment mais qui sont obligés, dans certaines circonstances, de s'allier pour fédérer les efforts et porter au plus haut leurs revendications. C'est le cas des revendications concernant les salaires de l'Etat ou la mécanisation des Enseignants. Il est vrai que chaque syndicat religieux a ses problèmes spécifiques liés à ses adhérents, mais ceci n'empêche qu'en cas de besoin ils ne puissent conjuguer les forces. 
Dans ce cas, la collaboration effective ne se réalise qu'entre dirigeants. Les syndicats chrétiens affichent cependant les limites dans les revendications en direction de leurs Eglises alors qu'elles se montrent efficaces dans les revendications en direction de l'Etat congolais. L'on constate souvent une mobilisation de grande envergure lorsque la revendication est adressée à l'Etat alors qu'il y a des réticences ou des hésitations chaque fois que la revendication s'adresse à l'Eglise. D'où la préférence exprimée par de nombreux travailleurs à l'endroit des syndicats neutres.

$\mathrm{Au}$ milieu de ces deux syndicats, s'interposent plusieurs autres syndicats privés non confessionnels réunissant les travailleurs de toutes tendances. A Bukavu, le cas le plus fréquent est celui du syndicat neutre, actif dans certaines professions comme l'Enseignement Supérieur et Universitaire (ESU), la coordination médicale et le secteur culturel avec l'Union Nationale des travailleurs du Congo (UNTC), la Convention démocratique du Travail (CDT), contrairement au syndicat chrétien qui est seulement en vogue et puissant dans l'enseignement primaire et secondaire. L'engouement vers le syndicat neutre à Bukavu est souvent justifié et encouragé par le respect de la justice et de l'équité ainsi que la liberté d'obéir à la conscience de chaque membre.

Le syndicat unique résulte du fait qu'un Etat peut imposer que les travailleurs réalisent l'unité syndicale comme c'était le cas de l'Union Nationale des Travailleurs du Zaïre (UNTZA). C'est de cette imposition des agents de s'unir dans un grand groupe que Léon XIII (1945) note que dans le syndicat unique, les chrétiens ont la responsabilité d'y faire pénétrer l'inspiration de l'Evangile, d'y exercer une influence et même, d'y devenir une force qui dirige et qui soutient comme un tuteur, l'activité syndicale.

Historiquement, le syndicat ouvrier est un organe de défense et de revendication dont les circonstances imposent la constitution et le développement. Au contraire, le syndicat patronal est un organe de gestion économique et sociale qui se développe lentement au gré des besoins.

Cette recherche fait constater qu'à Bukavu plusieurs organisations syndicales ou organisations professionnelles d'ouvriers, de médecins, d'agriculteurs, d'ingénieurs, de patrons et d'autres organisations similaires d'hommes et femmes, sont actives dans les mouvements de revendication.

Tableau 2. Test Khi-carré confession religieuse et existence de syndicat

\begin{tabular}{|l|c|c|c|}
\hline & Value & Df & Asymp. Sig. (2-sided) \\
\hline Pearson Chi-Square & 1.854 & 1 & .173 \\
Continuity Correction $^{\mathrm{b}}$ & 1.591 & 1 & .207 \\
Likelihood Ratio $_{\text {Linear-by-Linear }}^{1.855}$ & 1 & .173 \\
Association & 1.849 & 1 & .174 \\
N of Valid Cases & 401 & & \\
\hline
\end{tabular}

Source : Enquête de terrain, 2019 
Les résultats indiquent que les organisations à confession catholique sont les moins affiliées au syndicat, mais que la différence avec d'autres confessions n'est pas significative.

\subsection{Organisation du syndicat au sein des structures professionnelles des Eglises à Bukavu}

Dans l'entreprise, la vie est caractérisée par une série d'interactions des faits dans le rapport contractuel entre travailleur et employeur. L'action isolée du travailleur fragilise sa position vis-à-vis de l'employeur. Une action collective et concertée est plus efficace car dit-on, l'union fait la force. Pour amener les travailleurs à prendre collectivement conscience des problèmes qui sont les leurs dans l'entreprise et à décider de les résoudre, il faut qu'ils aient une organisation bien structurée. C'est dans ce cadre par exemple que les travailleurs du Bureau Diocésain des Euvres médicales (BDOM) se regroupent dans le Syndicat Libre Chrétien du Congo (SLCC) dont l'organigramme se présente comme suit.

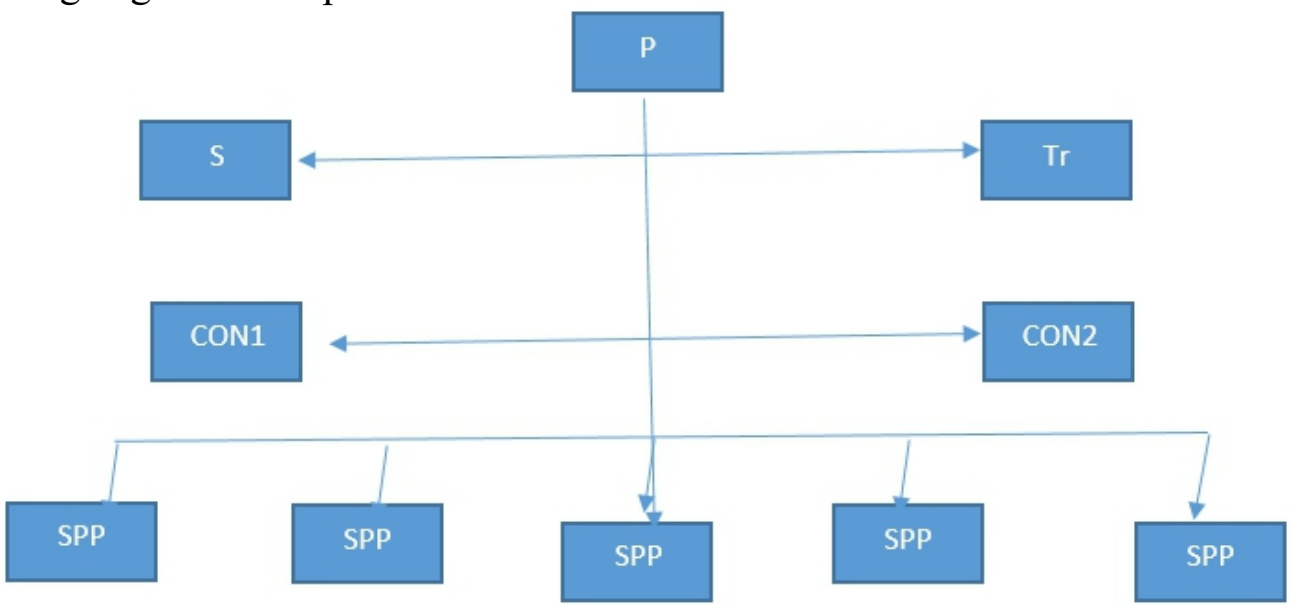

LEGENDE

Figure 1: Structure organique du syndicat au BDOM Bukavu.

$\mathrm{P}$ : président, $\mathrm{S}:$ secrétaire, Tr : Trésorier, CON1 : premier conseiller, CON2 : deuxième conseiller, SPP : Suppléant

Il ressort de cette figure que dans la délégation syndicale du BDOM est constituée de dix membres dont cinq effectifs et cinq suppléants. Les cinq membres effectifs forment le Bureau de la délégation syndicale qui du reste, est composé d'un Président, d'un Secrétaire, d'un Trésorier et de deux conseillers. Au BDOM comme dans toutes les entreprises de la RDC, la réunion de l'ensemble des membres constitue l'assemblée générale. L'AG est le souverain primaire. C'est le fondement de toutes les activités syndicales dans l'entreprise. Elle est la source de tout pouvoir syndical au sein du Bureau 
Diocésain des Euvres Médicales. Celle-ci produit par voie des élections un comité syndical dont la tâche est d'assurer la vie du syndicat dans l'entreprise.

\subsection{Mécanismes de revendication des syndicats}

Les négociations avec les employeurs constituent l'aspect désagréable ou douloureux de l'activité syndicale dans les structures professionnelles des Eglises. On le sait bien que c'est en fonction de la négociation qu'on prépare souvent et qu'on fait, quelquefois, la grève. En plus, c'est en vue de la négociation qu'on s'efforce de fortifier et d'unifier le syndicat. Les leaders tout comme les organisations sont également appréciés ou jugés sur la base de leurs résultats.

En dehors de la personne des négociateurs, la durée des conventions signées, la périodicité des négociations ont une importance capitale. Mais quelle est la procédure suivie par les agents pour exprimer leurs besoins ? Le tableau suivant permet de répondre à cette question.

Tableau 3. Relation confession religieuse et procédure de revendication des droits

\begin{tabular}{|l|l|l|l|}
\hline \multirow{2}{*}{$\begin{array}{l}\text { MECANISMES DE } \\
\text { REVENDICATION DES DROITS }\end{array}$} & \multicolumn{2}{|c|}{ CONFESSION RELIGIEUSE } & \multirow{2}{*}{ TOTAL } \\
\cline { 2 - 3 } & PROTESTANT & CATHOLIQUE & TOtre déposée à la délégation syndicale \\
\hline Lettre $69(34.7 \%)$ & $94(46.5 \%)$ & 163 \\
\hline $\begin{array}{l}\text { Lettre adressée à l'autorité religieuse de } \\
\text { la confession }\end{array}$ & $37(18.6 \%)$ & $69(34.1 \%)$ & 106 \\
\hline $\begin{array}{l}\text { Lettre déposée aux instances judiciaires } \\
\text { de la province }\end{array}$ & $21(10.5 \%)$ & 0 & 21 \\
\hline Le recours aux amis du chef & $36(18 \%)$ & $18(8.9 \%)$ & 54 \\
\hline L'affrontement direct du chef & $36(18 \%)$ & $21(10.4 \%)$ & 57 \\
\hline TOTAL & 199 & 202 & 401 \\
\hline
\end{tabular}

Source: Enquête de terrain, 2019

Le constat empirique relève qu'il existe plusieurs mécanismes de revendications des droits des travailleurs dans les services des Eglises à Bukavu. Le tableau démontre que 290 enquêtés privilégient les lettres adressées à des instances compétentes ou à la délégation syndicale dans le processus de revendication de leurs droits. Comme il a été dit plus haut dans cet article, la délégation syndicale est l'organe chargé de canaliser les revendications des travailleurs vers les autorités en vue des solutions. Il est évident que ces lettres sont adressées en majorité à la délégation syndicale néanmoins d'autres sont directement adressées à l'autorité religieuse sans passer par le syndicat. Cette sollicitation de l'autorité religieuse est souvent ambivalente. Elle peut intervenir favorablement ou défavorablement dans la résolution du problème. Mais dans un sens comme dans l'autre, le syndicat se voit étouffé car s'estimant le seul organe habileté à poser le problème ou à solliciter l'implication personnelle de l'instance supérieure ou religieuse. 
Cette attitude s'observe tantôt lorsque l'agent a déjà maintes fois posé son problème au syndicat sans y trouver solution; tantôt lorsque l'agent a une influence ou une relation personnelle avec l'autorité religieuse et qu'il estime devoir trouver solution facilement. Ainsi, le trafic d'influence a été constaté dans plusieurs services confessionnels à Bukavu. Les investigations attestent que certains responsables sont souvent poussés à la démission du fait d'avoir inquiété administrativement ou socialement les agents proches des autorités religieuses dans le service. A titre illustratif, frère cadet d'une autorité religieuse engagé dans la structure professionnelle confessionnelle est protégé et traité différemment d'un autre agent dont les relations sociales familiales ou amicales sont éloignées ou absentes.

Dans le même ordre d'idées, 54 agents, soit 13,4\%, procèdent par des recours auprès des amis de chef pour trouver solution à leurs problèmes. Comme on peut le constater, plusieurs agents survivent au sein des entreprises religieuses grâce au capital relationnel et ce, en dépit d'incompétence qu'ils affichent dans l'exercice de leurs fonctions. Ces agents soutenus ou ayant la couverture de l'autorité transgressent facilement les instructions qui leurs sont données par leurs chefs directs. Inversement, l'intervention de l'autorité religieuse est souvent salutaire dans la résolution de plusieurs problèmes au sein des entreprises des Eglises.

Dans la même perspective, vingt et une lettres, soit $5,2 \%$, sont adressées aux instances judiciaires notamment au juge et/ou à l'inspecteur du travail. Dans ce dernier cas, le dossier devient un litige professionnel qui ne peut plus trouver solution à l'interne. La présente recherche a identifié treize dossiers des litiges professionnels chez l'inspecteur du travail dont huit pour les employés catholiques (contre le Centre Olame, le Bureau Diocésain des Euvres Médicales, l'Economat général) et cinq dossiers des employés protestants (contre PMI, ZOA).

\subsection{Les activités syndicales des organisations professionnelles des Eglises}

L'objectif primordial de l'organisation professionnelle est en effet d'établir entre les membres d'une même profession des liens afin que tous, quelle que soit leur fonction dans la profession, poursuivent au-dessus de ces diversités et par elles, le bien commun de cette profession. L'intérêt général d'une profession demande qu'elle soit stable et prospère. Surtout le bien commun demande qu'une profession aide chacun de ses membres à exercer un métier qui les épanouisse et qu'elle établisse leurs rapports dans la justice et la collaboration loyale.

Les justes efforts pour défendre les droits des travailleurs unis dans la même profession doivent toujours tenir compte des limitations imposées par la situation économique générale du pays. La vie sociale et économico-sociale 
est certainement comme un système de « vases communicants » et chaque activité sociale, qui a pour but de sauvegarder les droits des groupes particuliers, doit s'y adapter.

Parlant de la sauvegarde des justes droits des travailleurs selon leurs diverses professions, il faut naturellement avoir toujours davantage devant les yeux ce dont dépend le caractère subjectif du travail dans chaque profession, mais en même temps ou avant tout, ce qui conditionne la dignité propre du sujet qui travaille. Ici, s'ouvrent de multiples possibilités pour l'action des organisations syndicales, y compris leur engagement en faveur de l'enseignement, de l'éducation et de la promotion de l'auto-éducation.

L'action des écoles, des programmes et des cours de formation qui ont développé et développent encore ce type d'activité, est bien apprécié par les enquêtés, membres des délégations syndicales. Le souhait est toujours que, grâce à l'action de son syndicat, le travailleur non seulement puisse « avoir » plus, mais aussi et surtout puisse « être » davantage, c'est-à-dire qu'il puisse réaliser plus pleinement son humanité sous tous ses aspects.

\section{IV.2. La perception sociale des fonctions remplies par les syndicats}

Le degré d'appréciation de l'action syndicale varie selon les acteurs agents des emplois des Eglises comme l'indique le tableau ci-après.

Tableau 4. Relation entre confession religieuse et appréciation des actions de la délégation syndicale

\begin{tabular}{|c|c|c|c|}
\hline \multirow[b]{2}{*}{ ACTIONS SYNDICALES } & \multicolumn{2}{|c|}{ CONFESSION RELIGIEUSE } & \multirow[b]{2}{*}{ TOTAL } \\
\hline & PROTESTANTE & CATHOLIQUE & \\
\hline Satisfaisantes & $44(38.9 \%)$ & $26(25.7 \%)$ & 70 \\
\hline Irrégulières & $9(8 \%)$ & $14(13.8)$ & 23 \\
\hline Décevantes & $18(15.9 \%)$ & $12(11.8)$ & 30 \\
\hline Inexistantes & $42(37.2 \%)$ & $49(48.5 \%)$ & 91 \\
\hline TOTAL & 113 & 101 & 214 \\
\hline
\end{tabular}

Source : Enquête de terrain, 2019

La lecture de ce tableau fait constater quatre différentes appréciations des agents consommateurs des actions syndicales. Ces actions sont ainsi qualifiées par les uns et les autres de satisfaisantes, d'irrégulières, décevantes et inexistantes. Ceux qui sont satisfaits des actions menées par leurs syndicats arguent que leurs syndicats interviennent au moment opportun, surtout en cas de besoin pour plaider et éventuellement remettre les agents dans leurs droits professionnels. Par contre, la catégorie des agents qui trouve les actions syndicales irrégulières dans leurs entreprises, s'appuie sur le fait que les syndicats n'interviennent qu'occasionnellement et seulement en faveur de certains dossiers. Dans le même ordre d'idées, les trente enquêtés qui jugent les actions syndicales décevantes soutiennent que les syndicats entreprennent 
beaucoup d'actions à la fois, mais qu'ils abandonnent en cours d'exécution causant ainsi le licenciement sans préavis de plusieurs agents. Enfin, un nombre important des agents trouve que les syndicats existent théoriquement dans leurs entreprises mais dans la pratique, ils n'exercent pas des activités.

\section{IV.3. Les contraintes des syndicats dans l'exercice de leurs missions.}

De ces résultats, il se dégage que les syndicats existent mais sont pour la plupart dysfonctionnels dans les services promus par les Eglises à Bukavu. Il n'est pas étonnant que le mouvement ouvrier se trouve nécessairement engagé dans deux rôles : l'un, de défense des salariés, l'autre de participation à la gestion indirecte de l'entreprise avec le chef. Parlant des entreprises sousdéveloppées et en situation de dépendance, cette recherche dégage quatre types des problèmes rencontrés sur le terrain. Il s'agit de :

1. appartenance à une confession religieuse qui entraine la soumission étroite à la logique du chef. L'on dirait que la plupart des délégués syndicaux issus des confessions religieuses peinent à se détacher de la philosophie des conditions de travail proposées par leurs Eglises.

Les délégués syndicaux sont ainsi contraints de travailler dans la logique des autorités investies par les Eglises.

Que la logique des revendications soit favorable ou pas au personnel, ce qui importe plus, c'est d'abord l'adhésion à la philosophie souhaitée et montée par les responsables des Eglises. Les agents sont ainsi invités à la sagesse et à protéger les intérêts de l'Eglise et les conditions sociales et économiques qui leur sont proposées par les services. Dans ce contexte caractérisé par la crise de l'emploi, les syndiqués s'accommodent et n'interviennent que rarement dans les actions de revendication.

Ceci fragilise la délégation syndicale qui ne peut pas intervenir, en dépit de sa compétence, sans le soutien de la base (les travailleurs) qui a la peur de perdre l'emploi. Dans le cas contraire, les employés se retrouvent dans une situation telle qu'on se met, soit à revendiquer avec tous les risques possibles d'être chassés, soit de démissionner en refusant de travailler sous les conditions précaires. D'autres employés sont déçus des actions syndicales qui sont sporadiques et toujours menées en faveur d'une catégorie des personnes. Certains délégués syndicaux sont taxés par les syndiqués lésés dans leurs droits d'être à la solde des Eglises ou de leurs frères suite à inaction. Dans le cas extrême, on retrouve des syndicats fictifs qui ne jouent plus leurs fonctions et qui se contentent à user des avantages liés à ce statut sans jouer les rôles correspondants. 
2. pauvreté et insuffisance des opportunités d'emplois constituent la deuxième faiblesse de la délégation syndicale : le mouvement social des travailleurs du secteur religieux est souvent bloqué par le manque des moyens financiers. Les travailleurs des Eglises, comme cela a été dit plus haut, n’ont pas des moyens financiers suffisants pour mener à bien leur mouvement. En effet, les cotisations au sein des entreprises confessionnelles varient entre 1 et 5 dollars américains. Ces contributions sont, soit retenues à la source avec bien évidemment la collaboration du responsable de l'entreprise, soit libérées à volonté par les syndiqués. Les affiliés occupant des emplois qui payent relativement moins (entre cinquante et cinq cents dollars américains, ne s'empêchent pas à se plaindre de l'argent cotisé sans résultat. Or, ne s'engagent dans le mouvement de défense des droits des travailleurs que les agents n'occupant pas des hautes fonctions de responsabilité au sein des Entreprises.

3. Motivations diversifiées des agents travaillant dans le secteur des Eglises : certains agents, au-delà des motivations économiques, sont également motivés spirituellement à travailler pour un salaire n'atteignant même pas le SMIG prévu au niveau national. Dans ce contexte, ce travail est un apostolat qu'ils rendent à leur Eglise. Ces agents sont plus nombreux dans le secteur professionnel paroissial ou des Eglises et ne trouvent pas souvent d'intérêts à adhérer à un mouvement de grève ou « sit in » organisé par la délégation syndicale de leur entreprise.

4. patronat composé essentiellement des responsables qui, euxmêmes sont membres de leurs Eglises. Ils ont été recrutés sur base des critères divers allant de l'objectivité à la subjectivité et ayant reçu des responsables des Eglises la mission de les assister dans la gestion d'un secteur professionnel précis. La subjectivité résulte du fait qu'au lieu que les recrutements se fondent sur la compétence et la transparence, elle se base sur le capital social, l'appartenance religieuse de certains chefs... Un chef recruté à base des critères subjectifs, ne laisse pas l'occasion aux agents de constituer un groupe fort et actif dans les revendications des agents. Il s'érige en obstacle à tout action tendant à fortifier ou à créer une cohésion entre les agents. A titre illustratif, les enquêtes ont relevé que les entreprises confessionnelles comme le Bureau Diocésain des Euvres médicales, la fondation Panzi et le Centre Heri Kwetu ont, maintes fois, étouffé les actions syndicales par crainte de la promotion du jeu démocratique à leur sein. En agissant pour les justes droits de leurs membres, les syndicats ont également recours au procédé de la "grève », c'est-à- 
dire de l'arrêt du travail conçu comme une sorte d'ultimatum adressé aux organes compétents et, avant tout, aux employeurs.

C'est un procédé que la doctrine sociale catholique reconnaît comme légitime sous certaines conditions (respect de service minimum, faciliter les négociations entre les parties) et dans de justes limites. Le principe de la liberté syndicale prévoit que les travailleurs devraient se voir assurer le droit de grève et ne pas subir de sanctions pénales personnelles pour leur participation à la grève. (Tajgman, D., 2000) Tout en admettant que celle-ci est un moyen juste et légitime, on doit également souligner qu'elle demeure, en un sens, un moyen extrême.

On ne peut pas en abuser; on ne peut pas en abuser spécialement pour faire le jeu de la politique. En outre, on ne peut jamais oublier que, lorsqu'il s'agit de services essentiels à la vie de la société, ces derniers doivent être toujours assurés, y compris, si c'est nécessaire, par des mesures légales adéquates. L'abus de la grève peut conduire à la paralysie de toute la vie socioéconomique. (Jean Paul II, 1981). Or cela est contraire aux exigences du bien commun de la société qui correspond également à la nature bien comprise du travail lui-même.

Les résultats indiquent que l'appréciation des actions syndicales diffère significativement selon que l'enquêté œuvre dans le service de la confession catholique ou dans le service de la confession protestante.

En effet, bien que dans les deux cas, le degré de satisfaction soit faible, les employés des confessions catholiques sont les plus déçus des actions de leur syndicat.

\section{Conclusion}

En cette période où la constitution de la RDC de 2006 consacre le pluralisme syndical, il y a lieu de s'interroger sur les différentes facettes de cette pratique dans les Entreprises et, plus particulièrement, dans les structures professionnelles promues par les Eglises de Bukavu. Le syndicat s'accompagne de la contestation violente de l'ordre établi et l'espérance, tout aussi violente, de transformer immédiatement le monde.

L'introduction du syndicat implique un bouleversement des relations existantes et un tel bouleversement suppose, dans les conditions culturelles de la ville de Bukavu, une idéologie révolutionnaire. La lutte pour améliorer la rémunération et les conditions matérielles du travail s'appuie sur cette contestation plus fondamentale qui donne bonne conscience au représentant ouvrier, oblige l'employeur à se tenir sur la défensive et fournit au leader syndical l'arme dont il se sert contre le patronat. Il peut l'utiliser dans un sens plus révolutionnaire ou plus réformiste. 
Au regard de ce constat, cet article s'est formulé au tour de trois objectifs notamment identifier les fonctions (les actions), jauger la perception sociale et dégager les contraintes des syndicats dans les structures professionnelles des Eglises. En vue d'atteindre ces objectifs, le recours à la méthode structuro-fonctionnaliste de Parsons et à la technique du questionnaire écrit ainsi qu'au logiciel SPSS N020 s'est avéré ind ispensable. Il est ressorti des recherches que : L'effectivité du mouvement syndical dans les structures professionnelles à Bukavu est constatée par 214 enquêtés, soit $53,3 \%$, reconnaissant la présence des syndicats au sein de leurs entreprises.

Cependant, les négociations avec les employeurs constituent l'aspect désagréable ou douloureux de l'activité du syndicalisme dans les structures professionnelles des Eglises. Les leaders tout comme les organisations sont également appréciés ou jugés sur base de leurs résultats. Certains enquêtés estiment que les actions des syndicats sont satisfaisantes dans leurs emplois alors que d'autres les trouvent décevantes. Il en résulte également que les syndicats existent mais sont pour la plupart dysfonctionnels dans les services promus par les Eglises à Bukavu.

Quant aux entreprises promues par les Eglises et en situation de dépendance, cet article a dégagé quatre types des problèmes rencontrés sur le terrain. Il s'agit de : l'appartenance à une confession religieuse qui pousse à l'adhésion à la philosophie souhaitée et montée par les responsables des Eglises, la pauvreté et l'insuffisance des opportunités d'emplois car le mouvement social des travailleurs du secteur religieux est souvent bloqué par le manque des moyens financiers, les motivations diversifiées des agents travaillant dans le secteur des Eglises : certains agents, au-delà des motivations économiques, sont également motivés spirituellement à travailler pour n'importe quel salaire et enfin, le patronat est composé essentiellement des responsables qui, eux-mêmes sont membres de leurs Eglises. Ces chefs ne laissent pas l'occasion aux agents de constituer un groupe fort et actif dans les revendications.

En marge de ce qui précède, cet article n'a pas la prétention d'avoir abordé tous les aspects liés au syndicalisme dans les structures professionnelles des Eglises à Bukavu. A titre d'exemple, il ne s'est pas attardé aux dispositions juridiques syndicales ni aux aspects idéologiques des représentations syndicales, il s'est juste limité à circonscrire les fonctions des syndicats, à jauger la perception sociale de leurs actions et à dégager les contraintes auxquelles ils font face dans la poursuite de leurs missions.

\section{References:}

1. Code Larcier (2003). Tome VI, R.D.Congo.

2. Constitution de la RDC du 18 février 2006. 
3. Cishunguluka, K. A. (2017). Entreprise coopérative d'épargne et de crédit : Lutte contre la pauvreté et pour la souveraineté sociale locale à Bukavu, Thèse de Doctorat, Université de Kisangani, Département de Sociologie, 119

4. Crozier, M. (1963). Le phénomène bureaucratique, Paris, Seuil, 13.

5. Division administrative de la mairie de Bukavu, Rapport annuel 2009

6. Division administrative de la mairie de Bukavu, Rapport annuel 2017

7. Droz, J. (1997). Histoire générale du socialisme, Paris, PUF, Quadrige, 19.

8. Fonteneau, G., Madounga, N. \& Linard, A. (2004). Histoire $d u$ syndicalisme en Afrique, Paris, Karthala, 111-113.

9. Friedmann, G. \& Naville, P. (1972). Traité de sociologie $d u$ travail, $3^{\text {ème }}$ édition, Paris, Armand Colin, 57.

10. Guerry, (Mgr), (1957). La Doctrine sociale de l'Eglise, Paris, 27.

11. Lecuyer, P., Boudon, R., Besnard, P., et Mohamed, C. (2003). Dictionnaire de sociologie, Larousse, Paris, VUEF, 51-53

12. Marx, K. (1965). Le Capital, Livre I, 1867, dans Euvres, Paris, Gallimard, La Pléiade, Tome 1, 79.

13. Ollandet, J., Histoire du mouvement syndical congolais, consulté le 16 juin 2014

14. Parsons, T. (1973). Sociétés. Essai sur leur évolution comparée (1996), Paris, Dunod, 36

15. Pape Jean Paul II. (1981). Laborem exercens, Kinshasa, Ed. Saint Paul Afrique, 49.

16. Pape Jean Paul II. (1983). Laberem exercens, Le travail, un bien pour l'homme. Ed. Presses universitaires de Namur, Namur.

17. Pape Léon XIII. (1945). Allocution aux travailleurs italiens,

18. Pape Léon XIII. (1891). Encyclique Rerum novarum, fr. m.wikipedia.org, consulté le 13 mai, 2016

19. Pape Pie X (1912), Encyclique singulari quadam, https//www.vatican, consulté le 21 mars 2010.

20. Pilo, K. P. \& Rugambwa, S. J. (2005) «Analyse critique des aspects historiques et écologiques de la ville de Bukavu et perspectives d'avenir », in Ujuvi, numero16, CRI, 11-39

21. Rollet, H. (1959). Le Travail, les Ouvriers et l'Eglise, Nihil Obstat, Paris, 12-18

22. Tajgman, D. (2000). Guide pratique de la liberté syndicale. Normes, principes et procédures de l'Organisation Internationale du Travail, Bureau international du Travail, Génève, 6-22 